\title{
High resolution bone material property assignment yields robust subject specific finite element models of complex thin bone structures
}

\author{
Pakdel A., Fialkov J., and Whyne C. M. \\ Version Post-Print/Accepted Manuscript \\ Citation Pakdel A, Fialkov J, Whyne CM. High resolution bone material \\ (published version) property assignment yields robust subject specific finite element models \\ of complex thin bone structures. J Biomech. 2016 Jun 14;49(9):145460. \\ doi: 10.1016/j.jbiomech.2016.03.015. PMID: 27033728
}

\section{Copyright / License (c) (i) $\ominus$}

This work is licensed under a Creative Commons AttributionNonCommercial-NoDerivatives 4.0 International License.

Publisher's Statement The final published version of this article is available at Elsevier via https://dx.doi.org/10.1016/j.jbiomech.2016.03.015.

Always cite the published version, so the author(s) will receive recognition through services that track citation counts, e.g. Scopus. If you need to cite the page number of the TSpace version (original manuscript or accepted manuscript) because you cannot access the published version, then cite the TSpace version in addition to the published version using the permanent URI (handle) found on the record page. 
Dear Author,

Please, note that changes made to the HTML content will be added to the article before publication, but are not reflected in this PDF.

Note also that this file should not be used for submitting corrections. 


\section{High resolution bone material property assignment yields robust subject specific finite element models of complex thin bone structures}

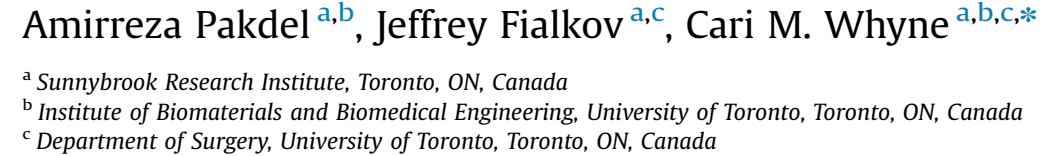

\section{A R T I C L E I N F O}

Article history:

Accepted 10 March 2016

\section{Keywords:}

Finite element analysis

Thin bone modeling

Craniomaxillofacial biomechanics

Bone material property assignment

Partial volume correction

\begin{abstract}
A B S T R A C T
Accurate finite element (FE) modeling of complex skeletal anatomy requires high resolution in both Q3 meshing and the heterogeneous mapping of material properties onto the generated mesh. This study introduces Node-based elastic Modulus Assignment with Partial-volume correction (NMAP) as a new approach for FE material property assignment to thin bone structures. The NMAP approach incorporates point spread function based deblurring of CT images, partial-volume correction of CT image voxel intensities and anisotropic interpolation and mapping of $C T$ intensity assignment to FE mesh nodes. The NMAP procedure combined with a derived craniomaxillo-facial skeleton (CMFS) specific densityisotropic elastic modulus relationship was applied to produce specimen-specific FE models of 6 cadaveric heads. The NMAP procedure successfully generated models of the complex thin bone structures with surface elastic moduli reflective of cortical bone material properties. The specimen-specific CMFS FE models were able to accurately predict experimental strains measured under in vitro temporalis and masseter muscle loading $(r=0.93$, slope $=1.01, n=5)$. The strength of this correlation represents a robust validation for CMFS FE modeling that can be used to better understand load transfer in this complex musculoskeletal system. The developed methodology offers a systematic process-flow able to address the complexity of the CMFS that can be further applied to create high-fidelity models of any musculoskeletal anatomy.
\end{abstract}

(c) 2016 Published by Elsevier Ltd.

\section{Introduction}

The most basic skeletal finite element (FE) models use homogeneous or limited heterogeneous segmentations to define bone material properties (Zannoni et al., 1998). Validated subjectspecific state-of-the-art FE studies have established that accurate skeletal models require both high resolution in meshing and heterogeneous material property mapping (Helgason et al., 2008b; Taddei et al., 2006; Schileo et al., 2007). Hounsfield units in a density calibrated computed tomography (CT) scanner can be correlated to bone mineral density values, followed by transformation to empirically derived elastic modulus values (Zannoni et al., 1998; Morgan et al., 2003; Keller, 1994; Dalstra et al., 1993; Carter and Hayes, 1977; Helgason et al., 2008a; Zioupos et al., 2008). The specific mathematical relationship between density

\footnotetext{
* Correspondence to: Orthopaedic Biomechanics Laboratory, S620, Sunnybrook Research Institute, 2075 Bayview Avenue, Toronto, Ontario, Canada M4N 3M5. Tel.: + 14164805056 .

E-mail address: cari.whyne@sunnybrook.ca (C.M. Whyne).
}

and modulus has been shown to be dependent on anatomical site and the density regime (cortical versus trabecular), however investigators have arrived at very different results even for bones of a single site and species (Schileo et al., 2007). This may be due to non-standard methods of measuring density and variable definitions of bone density, compounded by correlations used to convert from one definition to the other. Care must be taken in using extrapolations of phantom based measurements that relate trabecular intensity to density via linear interpolation and experimentally measured density to modulus correlations outside of experimentally validated bone density ranges. For density to modulus conversions specific to the craniomaxillo-facial skeleton (CMFS), Peterson et al. (Peterson and Dechow, 2003; Peterson et al., 2006) mapped the outer cortical table of the CMFS reporting regional anisotropic elastic properties, but the data set is too sparse to permit anisotropic mapping of elastic properties for a continuum CMFS mesh.

In long-bone skeletal biomechanics, the BONEMAT method has been most widely adopted and validated for subject-specific FE modeling (Viceconti et al., 2004; Poelert et al., 2012). This algorithm 

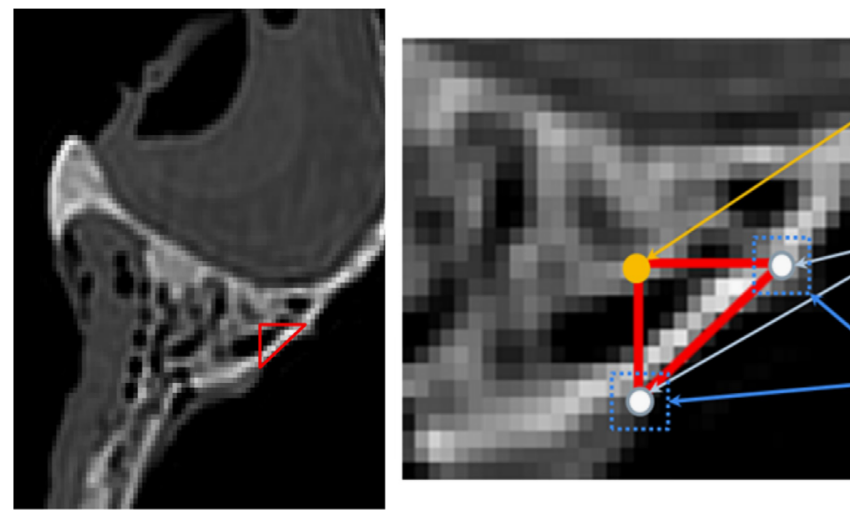

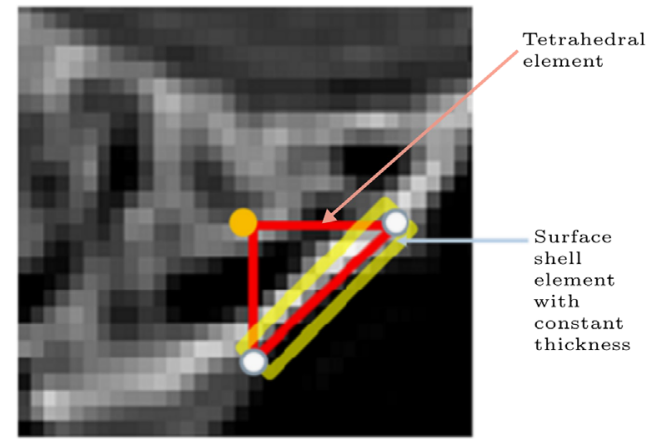

ounding

nodes
67

68

69

70

71

72

73

74

75

76

77

78

79

80

81

82

Fig. 1. An axial slice in the zygoma region. A cross-section of a tetrahedral mesh is illustrated in red. Close up of the element illustrates coordination of the nodes with the voxels on the CT images (middle) and the shell element approach for modeling of thin-bone structures (right). (For interpretation of the references to color in this figure legend, the reader is referred to the web version of this article.)
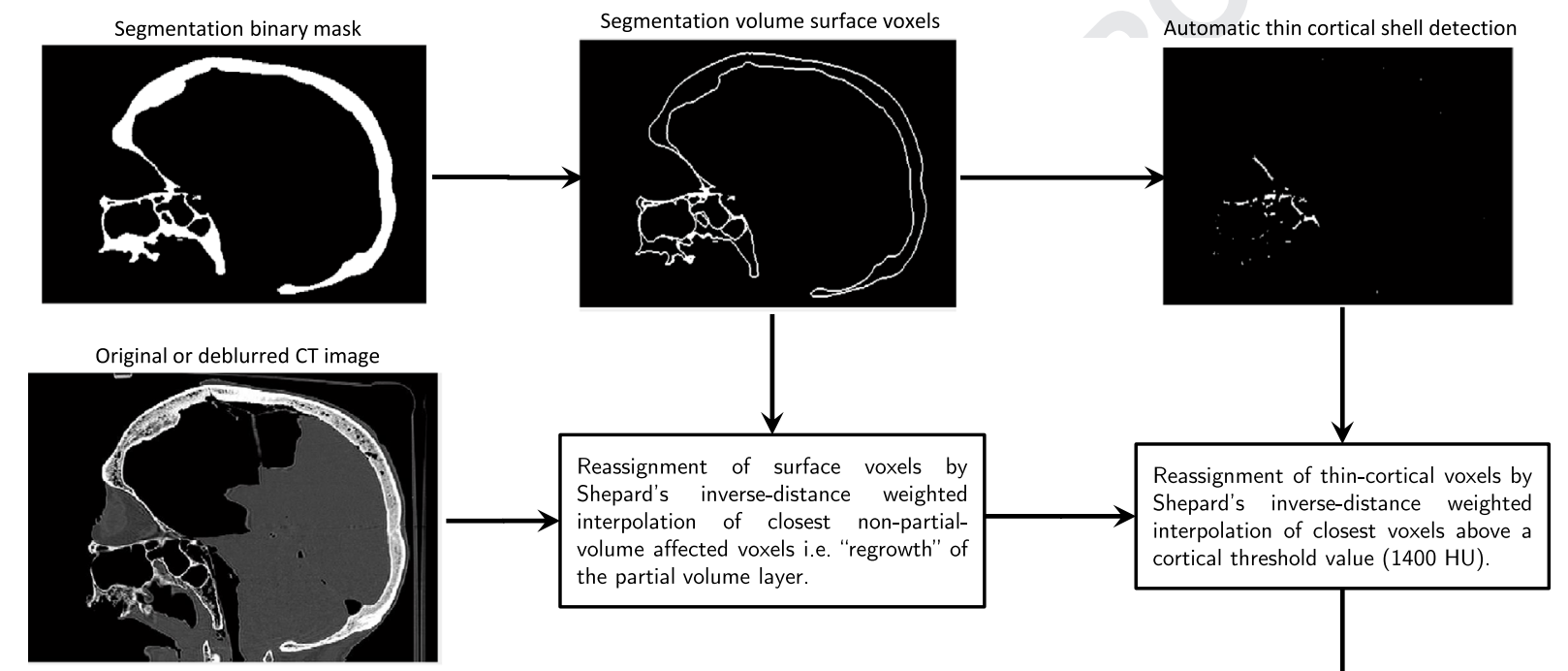
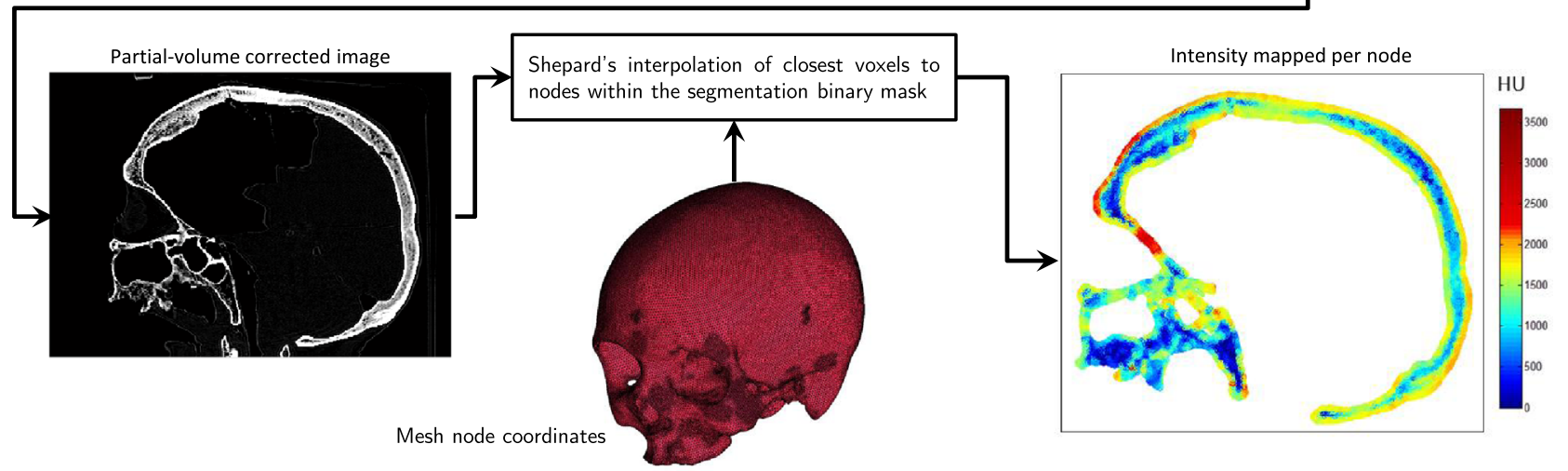

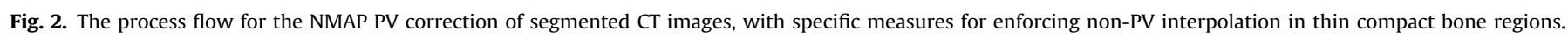

interpolates the intensity of the voxels mapped from the CT image to the volume of the elements of the FE mesh, assigning an elastic modulus to each individual element. Alternatively, voxels mapped to the vicinity of the nodes of each element can be assigned individual values (Helgason et al., 2008b). However, both surface nodes and elements may lie within regions which are corrupted by partial-volume (PV) effects. PV effects result in "blurring" of intensity values at boundaries with sharp intensity transitions, where the imaging system's resolution is unable to resolve the outline of thin, high-intensity structures (Ionescu et al., 2011). As shown in Fig. 1, materials of heterogenous intensities (from air to cortical bone) may be enclosed by a single element. The element's density, if calculated by averaging the enclosed voxels, results in a value equivalent to soft-tissue or low apparent density trabecular bone. To overcome this issue, some authors employ shell elements at the surface (Fig. 1, right), however, this simplification does not address the inability to sample and map the regional cortical bone density values on the FE mesh surfaces (Szwedowski et al., 2011; Anderson et al., 2005; Gupta et al., 2004).

Recent work has described the use of deblurring algorithms to reconstruct geometry and intensity values in CT data of skeletal structures (Pakdel et al., 2012, 2014). Deconvolution using a point spread function (PSF) has been shown to yield significant improvements in
83

84

85

86

87

88

89

90

91

92

93

94

95

96

97

98

99

100

101

102

103

104

105

106

107

108

109

110

111

112

113

114

115

116

117

118

119

120

121

122

123

124

125

126

127 


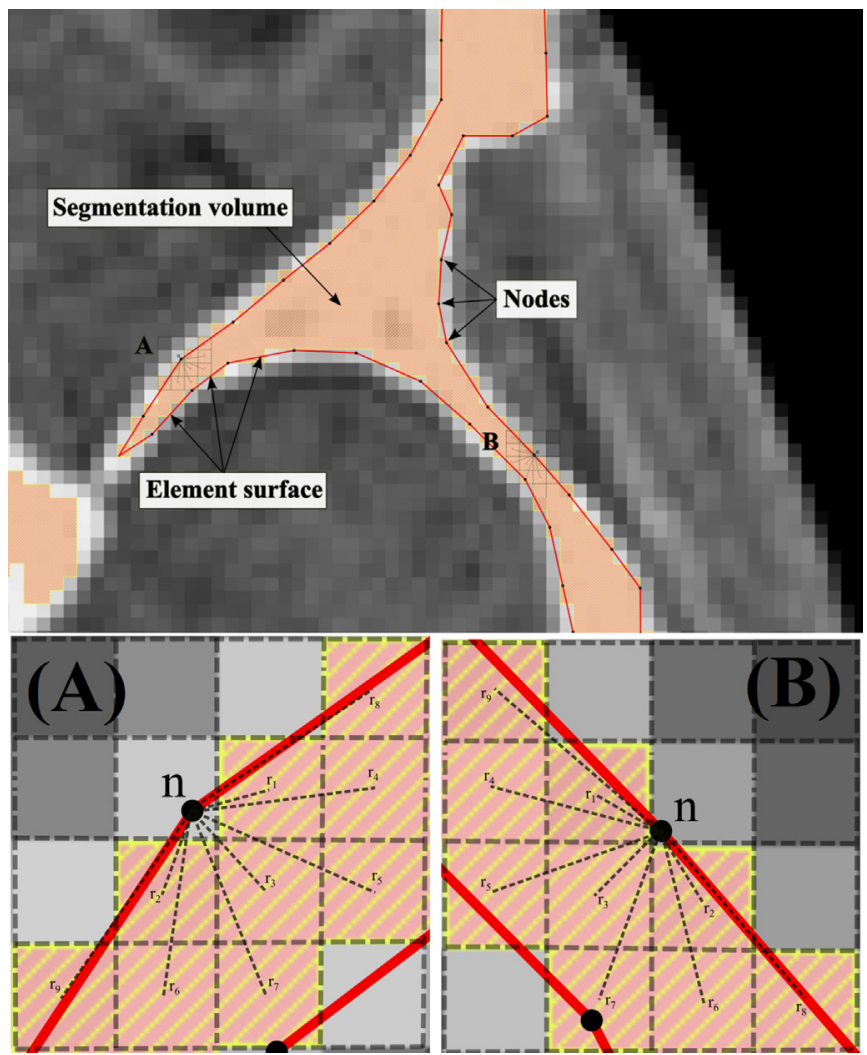

Fig. 3. Top: schematic illustration of node and element surface positions, superimposed on the CT image slice and the segmented bony region (orange area). Bottom row: close up of the annotated regions. The vectors $r_{i}$ represent the $i$ th closest voxel to the node $n$. This illustration is shown in 2D for simplicity; however the actual implementation is $3 \mathrm{D}$, and therefore the nodes' nearest voxels are not restricted to any slice plane. (For interpretation of the references to color in this figure legend, the reader is referred to the web version of this article.)

both geometry and intensity data for thin bone structures. While this deblurring approach can yield improved imaging data, loss of these benefits can occur through averaging of assigned material properties during FE meshing, as described above.

This study introduces a new methodology, Node-based elastic Modulus Assignment with Partial-volume correction (NMAP), for FE material property assignment which overcomes scaling and resolution limitations associated with existing element-based or node-based approaches. This paper applies the NMAP approach to the specific challenges of modeling the complex anatomy of the CMFS with its extensive network of thin bones, but the method constitutes a systematic process for ensuring the creation of models with high fidelity which can be applied to any skeletal anatomy. It was hypothesized that combining deblurring, nodebased elastic modulus assignment and partial volume correction will yield robust CMFS FE models able to predict experimental strains measured under in vitro muscle loading.

\section{Methods}

\subsection{Imaging}

Clinical CT scans were obtained from six male cadaveric CMFS specimens previously tested in vitro (Szwedowski et al., 2011; Maloul et al., 2012). One specimen was imaged at a voxel size of $0.523 \mathrm{~mm} \times 0.523 \mathrm{~mm} \times 0.8 \mathrm{~mm}$ (Philips Brilliance Big Bore, Philips, Amsterdam, Netherlands) with the other 5 imaged at a voxel size of $0.488 \mathrm{~mm} \times 0.488 \mathrm{~mm} \times 0.6 \mathrm{~mm}$ (GE LightSpeed VCT, GE Medical Systems, Waukesha, WI). The scans were acquired at $120 \mathrm{kVp}$ with an exposure of $215 \mathrm{mAs}$ and reconstructed using filtered back projection algorithms. The

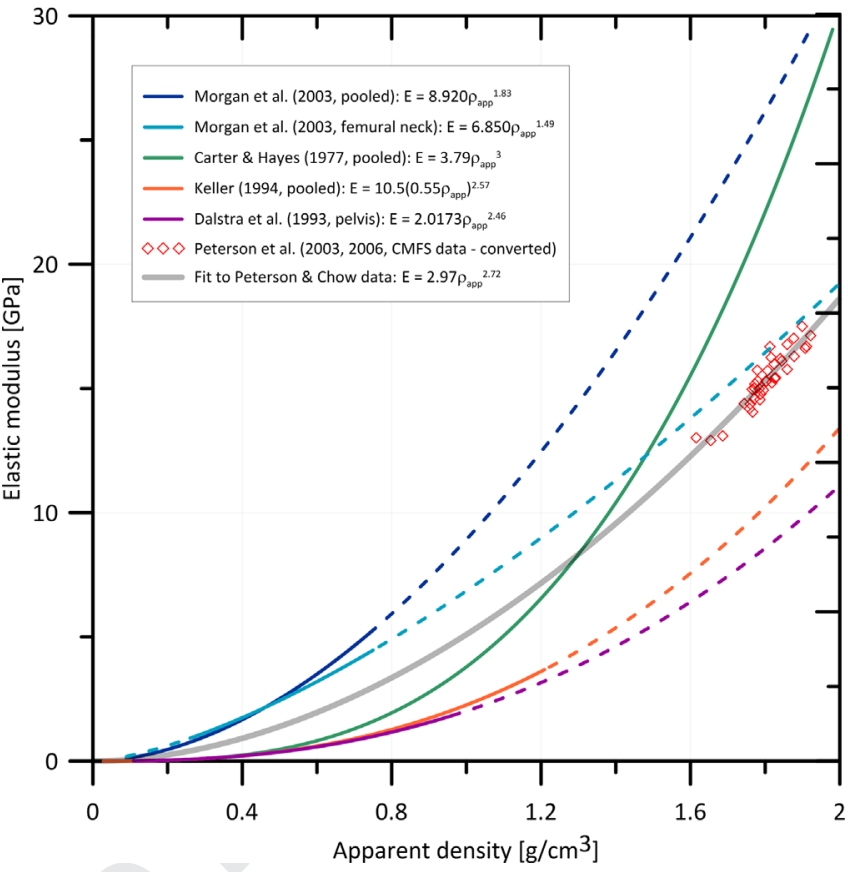

Fig. 4. A select number of empirical curves for conversion from apparent density to elastic modulus. The curves are plotted as solid lines and dashed lines, with the former representing the density range of the bone samples tested in the corresponding studies, and the latter indicating the extrapolation beyond these regions (Helgason et al., 2008a). The conversion applied on the Peterson et al. data is as described in the text. The best fit apparent density-modulus power relationship is included for this data. The Keller curve uses the conversion $\rho_{\text {app }}$ (apparent density in $\left.\mathrm{g} / \mathrm{cm}^{3}\right)=\rho_{\text {ash }}$ (ash density in $\left.\mathrm{g} / \mathrm{cm}^{3}\right) / 0.55$. The upper limit of cortical bone's apparent density is $\sim 1: 8 \mathrm{~g} / \mathrm{cm}^{3}$ (Carter and Hayes, 1977).

following methodology utilized these CT data sets to produce the subject-specific FE models.

2.2. FE model generation with node-based elastic modulus assignment with partialvolume correction (NMAP)

A sequential step-wise process for specimen specific FE model generation was employed using anisotropic Node-based elastic Modulus Assignment with Partialvolume correction (NMAP). The sequential steps of the NMAP procedure are summarized in Fig. 2 and described below.

\subsection{Deblurring of CT images with point-spread function (PSF)-estimation and deconvolution}

The 3D PSF were determined for each of the CT data sets and utilized within an iterative deconvolution algorithm to deblur the images in order to restore the geometrical details and intensity of high-contrast structures (bone) (Pakdel et al., 2012, 2014). This process has been demonstrated to improve both geometry and CT intensity in the CMFS. In this study, the MRNSD deconvolution algorithm (Bardsley and Nagy, 2006; Nagy and Strakos, 2000) was applied for 100 iterations for each sample prior to segmentation.

\subsection{Partial-volume correction of CT image voxel intensities}

The CT DICOM images and associated segmentation masks were loaded into MATLAB (MathWorks Inc., Natick, MA) as numerical matrices and the thickness of the partial-volume layer (PVL) to be corrected was determined. PVL refers to the number of voxel layers on the segmentation surface that are suspected to be corrupted by blurring. The main criterion to determine the PVL was the thickness of the smallest feature size that could be determined by thresholding (a minimum of 2 layers in this case). A 3-D implementation of the binary Euclidian Distance Transform (EDT) was performed on the segmentation mask to produce a distance map. The binary morphological operations of erosion, followed by dilation using a $3 \times 3$ binary kernel, were then applied on the segmentation mask to remove a 3-D boundary layer on the segmentation with a width equivalent to the PVL thickness. The subsequent dilation recovers all features except the thin cortical shells equal to or thinner than the PVL. This facilitates the selection of the thin cortical shells, which are stored in a separate segmentation mask. Based on the EDT 
image, all voxels whose distance from the surface were equal to or less than the PVL were removed.

The PVL can then be reconstructed based on statistical characteristics of the inner voxels which are presumed to be sufficiently far from the diffused boundaries as to not be PV-corrupted. The algorithm to "re-grow" (or re-grid) the voxels of the PVL is based on Sheppard's interpolation (Shepard, 1968). This procedure progresses layer by layer, beginning with the innermost of the removed layers. For each CT voxel in this initial layer, $N$ number of the closest voxels to it are found using k-nearest neighbors (kNN algorithm) (Friedman et al., 1977), and their distance and intensity values stored. The search is anisotropic by strict limitation of the search domain to all those voxels contained only within the segmentation mask, but not in the PVL that are not yet re-gridded. The value of that specific voxel is reassigned by Shepard's method given by

$H U(x, y, z)=\left[\sum_{i=0}^{N} \frac{\left(H U-H U_{i}\right)^{2}}{\left(r-r_{i}\right)^{p}}\right]^{1 / p}$

where the denominator term is the distance for each of the $N$ nearby voxels, and $p$ is the interpolation coefficient which controls the degree to which the radial distance is weighted in the interpolation. High values of $p$ demote the influence of faraway points, while $p$ values towards one effectively assign equal weight to all nearby voxels ( $N=25$ and $p=2$ were used in this study). Once an interpolated value is assigned, it is checked against its original value. If the value of the interpolation is lower than the original value, then the new interpolation is averaged with the previous value, and the average is assigned as the new value of the voxel. This process continues for all voxels in the layer. If the PVL variable value was greater than one, all the previous steps were repeated for the next layer(s). Note that the voxels that were interpolated in the inner layers are included in the kNN search space for the subsequent superficial layers. This is to ensure smoothness of the transition between all layers. Once all the PVL layers are completed, the procedure performs one final iteration for the thin-bone voxels (stored in the separate segmentation mask).

2.5. Anisotropic interpolation and mapping of CT intensity assignment on FE mesh nodes

This step assigns an interpolation value to each node of the FE mesh based on the PVcorrected image. Similar to the voxel re-gridding process, an interpolated Hounsfield units (HU) value is assigned to each node. First, the closest voxels to each node were found using the kNN search and limiting its search field to the domain of the segmentation mask. Subsequently, the interpolated $\mathrm{HU}$ value to be assigned to the node was calculated based on the distance of the closest voxels (Eq. (1), $p=5, N=6$ ). For comparison, the node-based material property assignment method described by Helgason et al. (2008b) 6 was also applied on one of the models (Fig. 3).
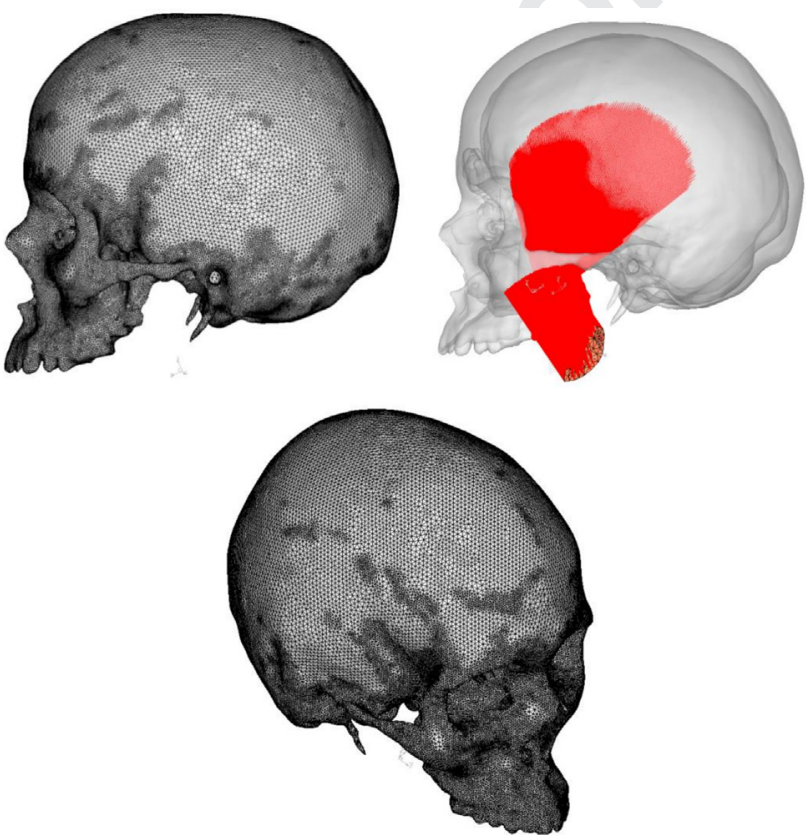

Fig. 5. The FE volume mesh and the constructed geometry representing the muscles on which loads were applied (masseter and temporalis, shown in red). These were modeled as tension-only link elements, originating from a digitized connective boundary to the digitized locations of the loading plates in the experiments. (For interpretation of the references to color in this figure legend, the reader is referred to the web version of this article.)

\subsection{Conversion of CT intensity to elastic modulus}

The HU to apparent density relationship was determined using bone-mineral phantoms in the clinical CT images (Wagner et al., 2011; Hangartner and Short 2007; Schweizer et al., 2007). However, there is an absence of reliable and validated empirical density to modulus conversions for the CMFS. The landmark contributions by Peterson et al. provide a mapping of the outer cortical table of the CMFS (Peterson and Dechow, 2003; Peterson et al., 2006). While their data is reported as sparse regional anisotropic elastic properties, and the sampling field is too sparse to be translated on a continuum for FE modeling, apparent density values were reported. In the current study, a CMFS-specific density-isotropic elastic modulus relationship was established based on the Peterson et al. data. The Voigt-ReussHill approach for estimating isotropic properties of bulk polycrystalline material by means of volumetric-average integration of the single crystal properties (Toonder et al., 1999) was used to adapt the Peterson et al. data to an estimated equivalent isotropic modulus. The derived apparent density to elastic modulus empirical conversion is shown in Fig. 4. The result most closely agrees with the densitymodulus relationship derived by Morgan et al. (2003) whose experimental data were based on femoral neck samples although at the given density range $\left(1.6<\rho<2 \mathrm{~g} / \mathrm{cm}^{3}\right)$ this relationship is largely extrapolated. The density in turn was converted to elastic modulus using the power relationship $E=2.97 \rho^{2.72}$ as determined by the fit for the Peterson and Chow data. The material property for each node was assigned using the temperature-dependent auxiliary material model as described in Helgason et al. (2008b) via a custom APDL macro. The use of the
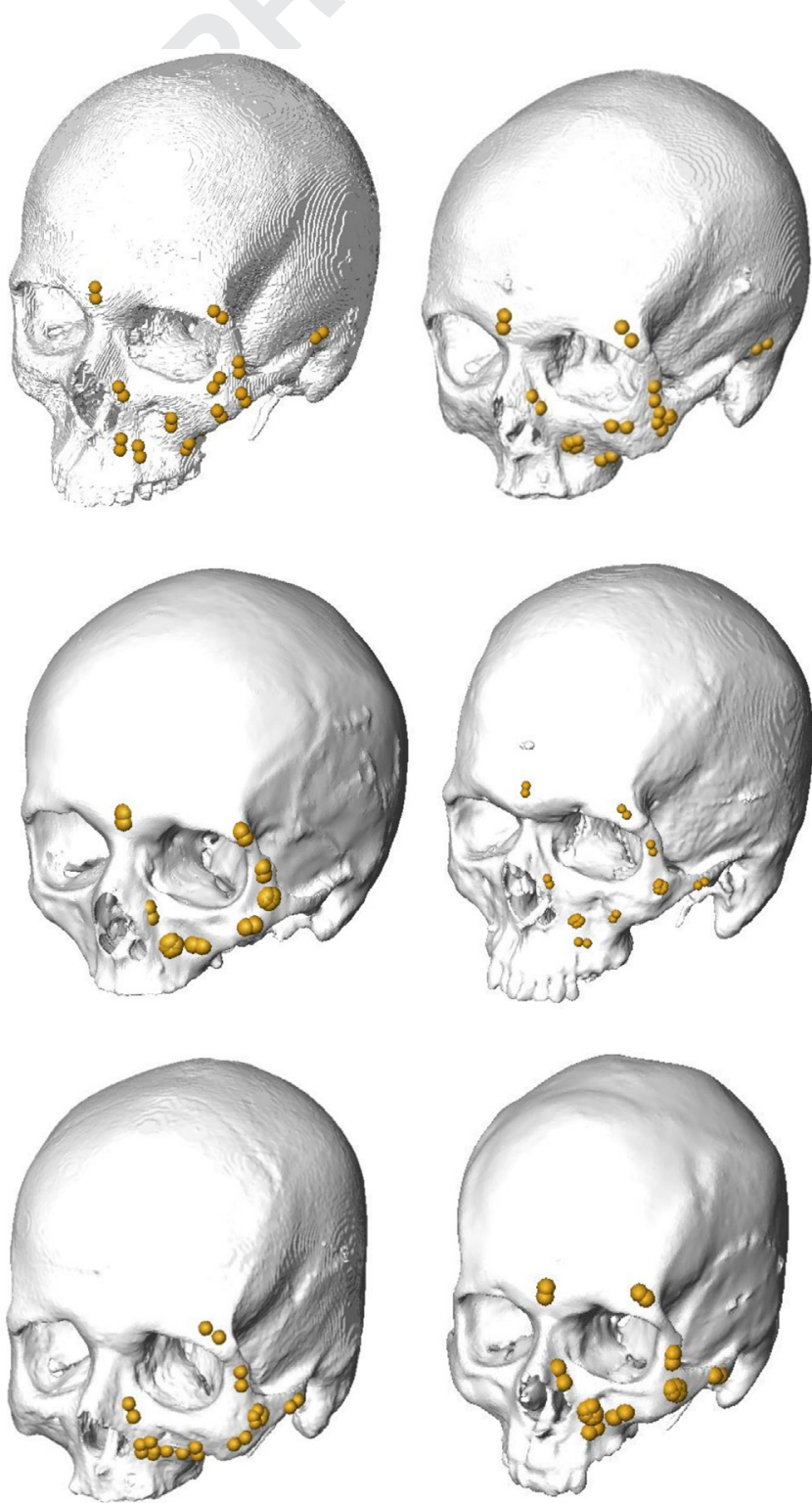

Fig. 6. Surface volumes of the subject-specific anatomies used for the validation studies, along with the digitized location of the strain gauges (spherical landmarks). 
temperature-dependency feature in ANSYS is applied solely to enable node based non-uniform elastic modulus assignment through a 1:1 correspondence between temperature and modulus. With the node-based approach utilized, elastic modulus within each volume is not constant, but rather linearly interpolated based on the value assigned to each of its nodes yielding a continuum of assigned elastic modulus values (rather than modulus confined to discrete bins as is the case with element based assignments)

\subsection{Load/boundary conditions and analysis}

Each FE mesh (quadratic 10-noded tetrahedral elements) was imported into and analyzed in ANSYS APDL (ANSYS Inc., Canonsburg, PA, USA). The boundary conditions were imposed to reflect the in vitro experimental conditions. Loading was applied independently through the masseter and temporalis muscles as specified by digitized coordinates and directionality of the experimental testing. For this, 3D spar elements (LINK 10) were used that are capable of representing uniaxial tension-only (Fig. 5). The isotropic tensile elements were assigned a modulus of $2 \mathrm{kPa}$ (Chen et al., 1996) and a Poisson's ratio of 0.35. There was no "adhesion"| coupling between adjacent elements, except where the elements shared nodes. The nodes at the loading plate were constrained to the plane of translation as digitized, with the final displacement screened to ensure the linear force vector as applied experimentally was followed. Full displacement degree-of-freedom constraints were applied to the outer surfaces of the teeth. The computational solution time was approximately eight hours for each multiple load case.

\subsection{Experimental in vitro strain data}

Surface strain data from the in vitro experiments previously conducted on the six cadaveric heads were used to validate the subject-specific FE models (Szwedowski et al. 2010, 2011; Maluoul et al., 2012). Briefly, the samples were fixed at the maxillary occlusion to an over-hang platform. An MTS Bionix 858 (MTS, Eden Prairie, Minnesota) servo-hydraulic loading system was used to apply controlled force vectors of 50,75 , and $100 \mathrm{~N}$ to the left masseter and temporalis muscles independently under displacement control at a rate of $1 \mathrm{~cm} / \mathrm{min}$. The strains generated for each test case were recorded on 14 strain gauges applied to the facial skeleton (Fig. 6). The coordinates of the strain gauges were digitized using a Microscribe digitizer (Immersion Corp., San Jose, California).

\subsection{FE post-processing and statistical validation}

The material properties and load/boundary conditions were applied in a consistent manner; no training algorithms were used for model optimization. Postanalysis, a custom APDL macro extracted the strain values on the mesh surface using landmark registrations and digitization coordinates with sub-mm precision. The strain values were calculated by interpolating the strain results within the elements which lay on a rectangular region equivalent to the bonded bounds of the strain gauges. This was done with respect to a transform coordinate that matched the strain's longitudinal axis. With most strain gauges the surface of interpolation had to be registered against the bony curvature. The strain interpolation was thus iterated and averaged 5 times, where each iteration penetrated the surface in $0.1 \mathrm{~mm}$ increments. The strain correlation results comparing the FE and experimental data were analyzed with standard bivariate models using JMP (4.0, SAS Institute, Cary, NC).

\section{Results}

The six FE models of the CMFS were successfully generated using the described methodology. The range of the assigned apparent densities and elastic modulus values compared positively to directly measured values reported by Peterson (Peterson and Dechow, 2003; Peterson et al., 2006). The output of the NMAP processing for one FE mesh is shown in Fig. 7 (top and middle). Fig. 7 (bottom) illustrates the nodal mapping results using the algorithm outlined by Helgason et al. (2008b). The material properties assigned to the surface elements are greatly impacted by PV effects, resulting in low modulus values not representative of cortical bone with standard node-based assignment. In contrast, the NMAP results yield surface HU and modulus values characteristic of cortical bone.

The experimental strain data from the in vitro testing of these six heads were previously published (Maloul et al., 2012; Szwedowski et al., 2010, 2011). After reviewing the trajectory of the

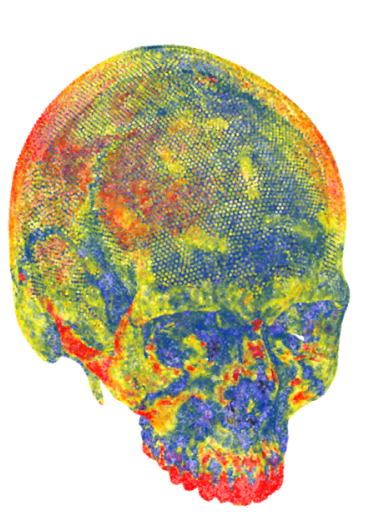

NMAP
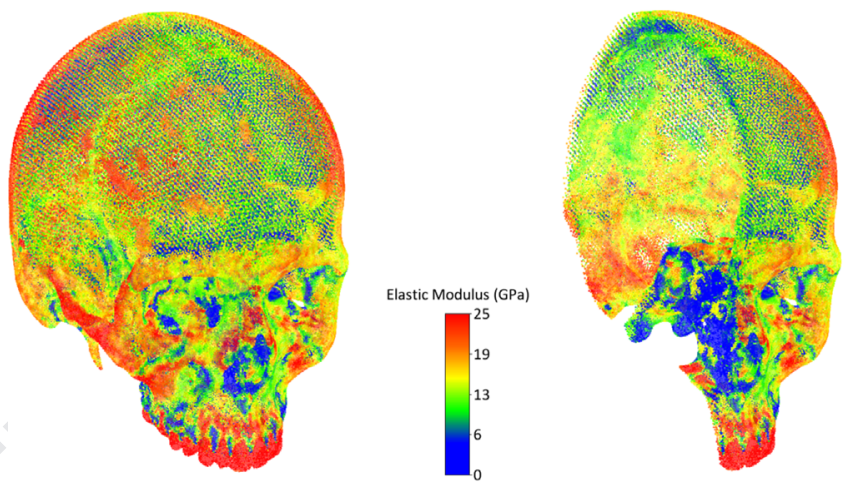

Standardnode-basedassignment
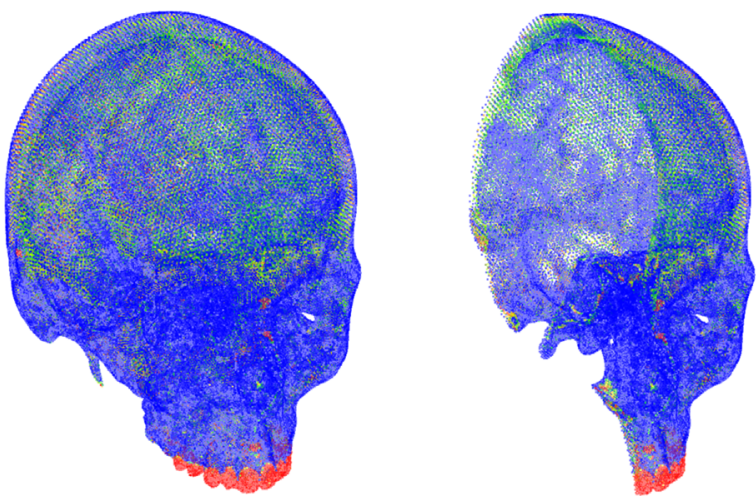

Fig. 7. Example results of the NMAP procedure. Each plot point is a corner node from an element in the mesh. Top row: the FE mesh visualized as color clusters of the HU values assigned to each node. Middle row: the conversion of HU values at the nodes to elastic modulus. Bottom row: elastic modulus values calculated and assigned using the algorithm of Helgason et al. (2008b). The color scale is the same as above. Extensive PV degradation has produced suppressed modulus values on the superficial nodes. Right column: cross-section view, showing mapping on the inner structure of the sphenoid and paranasal cavities. (For interpretation of the references to color in this figure legend, the reader is referred to the web version of this article.)

screw fixations that were used to stabilize the maxillary occlusion of one edentulous sample, it was discovered that screws penetrated intricate internal structures. An appropriate FE boundary condition could not be produced to represent this damage. As such, this model was excluded, leaving a sample size of five for further analysis.

The orthogonal regression analysis, shown in Fig. 8, summarizes the data from all specimens and loading conditions. Overall, a correlation coefficient of 0.93 was found comparing the FE model strains and the experimental data, $\left(r^{2}=0.87\right.$, slope $=1.01$, intercept $=-8.57$, power $=0.81, \alpha=0.05$ ) (Cohen, 2013). The high correlation coefficient and slope close to unity, confirm the
111 


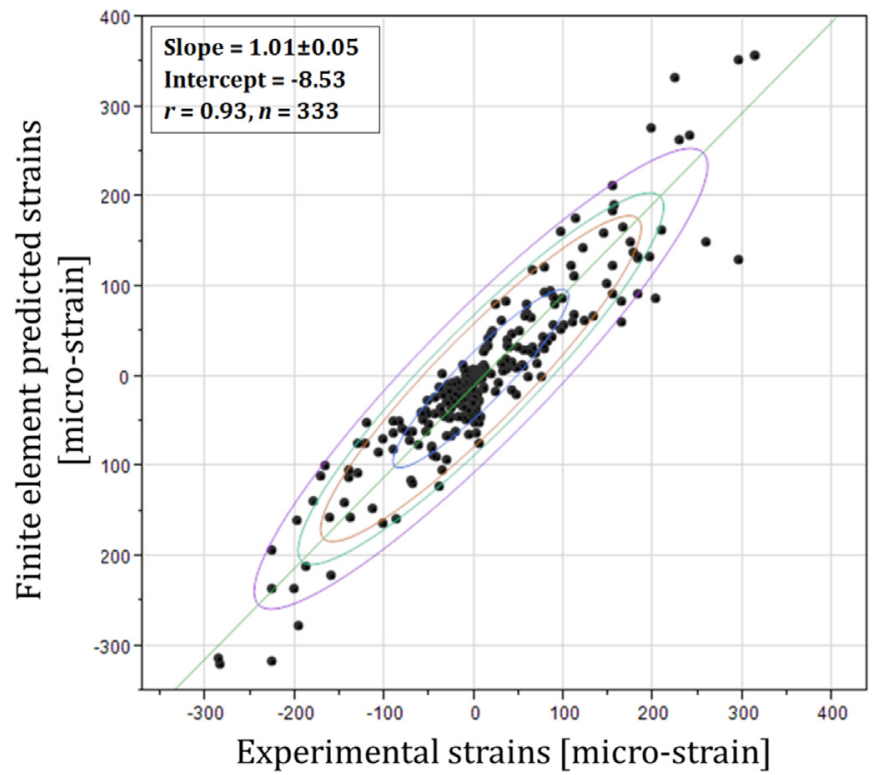

Fig. 8. : Orthogonal regression analysis of the aggregate of all the FE predicted strains correlated with the strains measured in vitro, inclusive of all the loading conditions. The concentric circles outline the bivariate normal (density) ellipses at $0.5,0.9,0.95$, and 0.99 confidence intervals increasing from the inner ellipse outwards.

hypothesis that the FE models generated using the NMAP procedure can accurately predict CMFS strains under in vitro conditions with applied simulated muscle loading.

\section{Discussion}

Robust geometrical models were derived from accurate secondary reconstruction of clinical CT images, which along with a higher resolution heterogeneous mapping of material properties of the CMFS yielded strong a correlation between the strain predicted from the subject-specific FE models to in vitro experimentally measured strains. The developed methodology offers a systematic process-flow to address the once intractable complexity of the CMFS, towards a robust biomechanical framework for understanding its structure and function. The strength of the correlations and the confidence intervals reported in this study represent the most robust validation results reported for CMFS FE modeling to date.

A previous FE validation and sensitivity study exists for the data from one CMFS used in this analysis. This FE model utilized differentially segmented cortical and trabecular regions of the CMFS, and assignment of a constant cortical bone value to the outer surface defined by a variable thickness shell element layer (Szwedowski et al., 2011). CT based material properties for the trabecular centrum were applied using BONEMAT. The optimized model configuration based on the sensitivity study yielded a good correlation to the experimental data $\left(r^{2}=0.71\right.$, slope $\left.=0.82\right)$. In this, the lowest correlation regions were found within the vicinity of thin bones that could not be accurately segmented and meshed. The current model using the developed approach, yielded a significantly better correlation to the experimental data $\left(r^{2}=0.85\right.$, slope $=1$ ) without differentiation of the cortical and trabecular bone or the use of shell elements (transformed $z$-test for comparing the inequality of two dependent Pearson r's, $\alpha=0.01$ (Steiger, 1980; Dunn and clark, 1969). The shell-based approach is more complex than assigning volumetrically distributed material properties to a continuum mesh, and does not address the inherent issues of degraded bone intensity signal in very thin bones. The shell-element approach also demands segmentation of cortical regions, which is particularly challenging in the context of the complex CMFS morphology.

The generation of CMFS models from specimens that include soft tissue structures have challenges not found in defleshed or dried anatomic specimens. PV degradation and low tissue contrast is less pronounced, creating sharp boundaries for straightforward segmentation from surrounding air. In contrast, muscle-fat-thinbone ambiguity pervades in full head cadaveric CT imaging. This may explain the unsuccessful application of previous nodal based material property assignment methods to CMFS cadavers, as demonstrated in Fig. 7. In contrast, the resolution of material properties achieved by the NMAP process is even able to capture the fine variation of densities near the sutures in the FE mesh (top row of Fig. 7). While material properties mapped onto the FE mesh of larger load bearing bones are not immune from suppression of surface CT intensity values, the total ratio of the PV degraded region relative to the overall cortical bone mass is small, limiting its impact (Austman et al., 2008). In contrast, even the thickest cranial bones can have up to $20 \%$ of their cortical region degraded by PV effects.

The NMAP procedure can be applied without deblurring, but in clinical CT reconstructions the presence of large holes in thin bone structures and the low moduli of blurred edges cannot be rectified with PV correction alone. NMAP may be successfully applied to higher resolution (i.e. $\mu \mathrm{CT}$ ) data sets or thicker cortical structures without deblurring.

While no intra-subject segmentation sensitivity analyses were conducted, consistently robust results were achieved for the multiple specimens using this methodological approach. The results of the PVL depend on the variables chosen for Sheppard's algorithm. The weighting of the proximal voxels (p) and the number of voxels $(N)$ were chosen as a best-fit to yield a balance of correction without over-smoothing. Future work may investigate the impact of optimization on these parameters with final validation on an independent set of samples.

The experimental approach in this study was designed for validation, rather than reflecting a physiologic scenario. The loading, while applied through individual muscles directly pulled in a controlled vector, does not reflect the complexity of masseter and temporalis function in vivo. Physiologic loading includes changes in the overall length, shape, and activation patterns in multiple masticatory muscles acting in concert (Goto et al., 2001). The non-pathological masticatory system also activates the muscles on both sides of the face for all masticatory functions at equivalent levels (regardless of ipsilateral, contralateral, or any other mode of chewing) and engages multiple muscle groups simultaneously, including neck muscles for stabilization during jaw activity (Van Eijden et al., 1990; Hellmann et al., 2012). The maxillary occlusion boundary condition represented in this validation work was also not physiological, but rather represented an experimentally feasible testing method.

In conclusion, the contribution of this study is a fully developed and validated subject-specific computational framework for deployment towards "in silico" investigation of CMFS biomechanics (Westwood, 2007) and a set of validated CMFS FE models. Future work may utilize these validated models under more physiologic load and boundary conditions to better understand load transmission patterns in the CMFS and employ this approach to the modeling of other thin bone skeletal structures.

\section{Conflict of interest}

There are no conflicts of interest with regards to the preparation and production of this research. 
7

9

10

\section{Acknowledgments}

Support for this work was provided by the Natural Sciences and 4 Q9 Engineering Research Council of Canada (NSERC \#RGPIN2392065010 11) and the Ontario Graduate Scholarship Program.

Morgan, E.F., Bayraktar, H.H., Keaveny, T.M., 2003. Trabecular bone modulusdensity relationships depend on anatomic site. J. Biomech. 36 (7), 897-904.

Nagy, J.G., Strakos, Z., 2000. Enforcing nonnegativity in image reconstruction algorithms. In: International Symposium on Optical Science and Technology. Int. Soc. Opt. Photonics, 182-190.

Pakdel, A., Robert, N., Fialkov, J., Maloul, A., Whyne, C., 2012. Generalized method for computation of true thickness and X-ray intensity information in highly blurred sub-millimeter bone features in clinical CT images. Phys. Med. Biol. 57 (23), 8099-8116

\section{References}

Anderson, A.E., Peters, C.L., Tuttle, B.D., Weiss, J.A., 2005. Subject-specific finite element model of the pelvis: development, validation and sensitivity studies. J. Biomech. Eng. 127 (3), 364.

Austman, R.L., Milner, J.S., Holdsworth, D.W., Dunning, C.E., 2008. The effect of the density-modulus relationship selected to apply material properties in a finite element model of long bone. J. Biomech. 41 (15), 3171-3176.

Bardsley, J., Nagy, J., 2006. Covariance-preconditioned iterative methods for nonnegatively constrained astronomical imaging. SIAM J. Matrix Anal. Appl. 27 (4), $1184-1197$.

Carter, D.R., Hayes, W.C., 1977. The compressive behavior of bone as a two-phase porous structure. J. Bone Jt. Surg. Am. Vol. 59 (7), 954-962.

Chen, E.J., Novakofski, J., Jenkins, W.K., O'Brien, W., 1996. Young's modulus measurements of soft tissues with application to elasticity imaging. IEEE Trans. Ultrason. Ferroelectr. Freq. Control 43 (1), 191-194.

Cohen, J., 2013. Statistical Power Analysis for the Behavioral Sciences, Vol. 2nd. Routledge.

Dalstra, M., Huiskes, R., Odgaard, A., Van Erning, L., 1993. Mechanical and textura properties of pelvic trabecular bone. J. Biomech. 26, 4.

Dunn, O.J., Mar, Clark V., 1969. Correlation coefficients measured on the same individuals. J. Am. Stat. Assoc. 64 (325), 366.

Friedman, J., Bentley, J., Finkel, R., 1977. An algorithm for finding best matches in logarithmic expected time. ACM Trans. Math. Softw. 3 (3), 209-226.

Goto, T.K.T., Langenbach, G.E.J., Hannam, A.G., 2001. Length changes in the human masseter muscle after jaw movement. Anat. Rec. 262 (3), 293-300.

Gupta, S., van der Helm, F.C.T. Sterk, J.C. van Keulen, F. Kaptein, B.L. 2004 Development and experimental validation of a three-dimensional finite element model of the human scapula. Proc. Inst. Mech. Eng. Part H: J. Eng. Med. 218 (2), 127-142.

Hangartner, T.N., Short, D.F., 2007. Accurate quantification of width and density of bone structures by computed tomography. Med. Phys. 34 (10), 3777.

Helgason, B., Perilli, E., Schileo, E., Taddei, F., Brynjolfsson, S., Viceconti, M., 2008a. Mathematical relationships between bone density and mechanical properties: a literature review. Clin. Biomech. 23 (2), 135-146.

Helgason, B., Taddei, F., P_alsson, H., Schileo, E., Cristofolini, L., Viceconti, M., Brynjolfsson, S., 2008b. A modified method for assigning material properties to FE models of bones. Med. Eng. Phys. 30 (4), 444-453.

Hellmann, D., Giannakopoulos, N.N., Schmitter, M., Lenz, J., Schindler, H.J., 2012. Anterior and posterior neck muscle activation during a variety of biting tasks. Eur. J. Oral Sci. 120 (4), 326-334.

Ionescu, M., Metcalfe, R.W., Cody, D., Alvarado, M.V.Y., Hipp, J., Benndorf, G., 2011. Spatial resolution limits of multislice computed tomography (MS-CT), C-arm$\mathrm{CT}$, and at panel-CT (FP-CT) compared to MicroCT for visualization of a small metallic stent. Acad. Radiol. 18 (7), 866-875.

Keller, T.S., 1994. Predicting the compressive mechanical behavior of bone. J. Biomech. 27 (9), 1159-1168.

Maloul, A., Regev, E., Whyne, C.M., Beek, M., Fialkov, J.A., 2012. In vitro quantifcation of strain patterns in the craniofacial skeleton due to masseter and temporalis activities. J. Craniofac. Surg. 23 (5), 1529-1534.
Pakdel, A., Mainprize, J.G., Robert, N., Fialkov, J., Whyne, C.M., 2014. Model-based PSF and MTF estimation and validation from skeletal clinical CT images. Med. Phys. 41 (1), 011906

Peterson, J., Dechow, P.C., 2003. Material properties of the human cranial vault and

Peterson, J., Wang, Q., Dechow, P.C., 2006. Material properties of the dentate maxilla. Anat. Rec. Part A: Discov. Mol. Cell. Evolut. Biol. 288 (9), 962-972.

Poelert, S., Valstar, E., Weinans, H., Zadpoor, A.A., 2012. Patient-specific finite element modeling of bones. Proc. Inst. Mech. Eng. Part H: J. Eng. Med. 227 (4), 464-478.

Schileo, E., Taddei, F., Malandrino, A., Cristofolini, L., Viceconti, M., 2007. Subjectspecific finite element models can accurately predict strain levels in long bones. J. Biomech. 40 (13), 2982-2989.

Schweizer, S., Hattendorf, B., Schneider, P., Aeschlimann, B., Gauckler, L., Muller, R., Gunther, D., 2007. Preparation and characterization of calibration standards for bone density determination by micro-computed tomography. Analyst 132 (10), 1040-1045.

Shepard, D., 1968. A two-dimensional interpolation function for irregularly-spaced data. In: Proceedings of the 23rd ACM National Conference, pp. 517-524.

Steiger, J.H., 1980. Tests for comparing elements of a correlation matrix. Psychol. Bull. 87 (2), 245-251.

Szwedowski, T.D., Fialkov, J., Whyne, C.M., 2011. Sensitivity analysis of a validated subject-specific finite element model of the human craniofacial skeleton. Proc. Inst. Mech. Eng. Part H: J. Eng. Med. 225 (1), 58-67.

Szwedowski, T.D., Whyne, C.M., Fialkov, J. a, 2010. Toward characterization of craniofacial biomechanics. J. Craniofac. Surg. 21 (1), 202-207.

Taddei, F., Martelli, S., Reggiani, B., Cristofolini, L., Viceconti, M., 2006. Finiteelement modeling of bones from CT data: sensitivity to geometry and material uncertainties. IEEE Trans. Bio-Med. Eng. 53 (11), 2194-2200.

Toonder, J.M.J.D., Dommelen, J. a W.V., Baaijens, F.P.T., 1999. The relation between single crystal elasticity and the effective elastic behaviour of polycrystalline materials: theory, measurement and computation. Model. Simul. Mater. Sci. Eng. 7 (6), 909-928.

Van Eijden, T.M., Brugman, P., Weijs, W. a, Oosting, J., 1990. Coactivation of jaw muscles: recruitment order and level as a function of bite force direction and magnitude. J. Biomech. 23 (5), 475-485.

Viceconti, M., Davinelli, M., Taddei, F., Cappello, A., 2004. Automatic generation of accurate subject-specific bone finite element models to be used in clinical studies. J. Biomech. 37 (10), 1597-1605.

Wagner, D.W., Lindsey, D.P., Beaupre, G.S., 2011. Deriving tissue density and elastic modulus from microCT bone scans. Bone 49 (5), 931-938.

Westwood, J.D., 2007. Medicine Meets Virtual Reality 15: In Vivo, In Vitro, In Silico: Designing the Next in Medicine. IOS Press.

Zannoni, C., Mantovani, R., Viceconti, M., 1998. Material properties assignment to finite element models of bone structures: a new method. Med. Eng. Phys. 20 (10), 735-740.

Zioupos, P., Cook, R.B., Hutchinson, J.R., 2008. Some basic relationships between density values in cancellous and cortical bone. J. Biomech. 41 (9), 1961. zygoma. Anat. Rec. Part A: Discov. Mol. Cell. Evolut. Biol. 274 (1), 785-797. 